\title{
Ken HYLAND
}

\section{Disciplinary Identities. Individuality and community in academic discourse Cambridge: Cambridge University Press.}

2012, 236 páginas.

ISBN: 9780521197595

La identidad ha ido constituyéndose en un concepto cada vez más visitado por investigadores de distintas áreas de las humanidades y las ciencias sociales, en particular. La creciente atención que ha suscitado, en los últimos veinticinco años, principalmente, ha derivado en la formulación de un sinfín de definiciones acerca de lo que encierra la noción de identidad, en estricto rigor. De este modo, las múltiples perspectivas desde las cuales se la ha examinado no hacen más que confirmar la relevancia de este bullente campo de investigación. En el libro que a continuación reseñamos, es el espacio académico el lugar dentro del cual se inserta la discusión en torno a la identidad. Su autor, Ken Hyland, sostiene que la universidad ha sido un contexto dentro del cual se ha descuidado el interés por la construcción discursiva de la identidad. A través de nueve capítulos, se propone revisar los rasgos que dan forma a la escritura académica y, principalmente, el modo mediante el cual la identidad está implicada en ella. En otras palabras, este libro explora el significado de la identidad académica, el cómo esta es construida por individuos que van conformando discursos que, en definitiva, los conectan con sus propias disciplinas.

El capítulo uno ("Identity: Interaction and community") revisa algunas de las principales ideas en torno al rasgo interaccional $-\mathrm{y}$, en consecuencia, social- que posee la identidad. De manera simple, la identidad es quién y qué somos; la forma en que los individuos experimentan y manejan el sentido de sí mismos, en tanto, es una cuestión más compleja. Esto, porque, como señala Hyland, las personas tienden a verse a sí mismas como individuos únicos que portan una especie de esencia y que, por ende, poseen una identidad estanca. No obstante ello, también se reconoce que nuestros comportamientos, nuestras filiaciones e, incluso, la forma en que hablamos cambian a través de las interacciones con diferentes personas, en contextos particulares, lo que, a menudo, causa algunas tensiones y conflictos. Para desarrollar estos planteamientos, el autor delimita tres subtópicos, a saber, (1) la identidad y la interacción, (2) la identidad y la comunidad y (3) la identidad y el discurso académico. En (1) se menciona la profunda suspicacia con que algunas teorías de corte postestructuralista han evaluado la noción contenida en el clásico aforismo cartesiano Pienso, luego existo, sobre todo, en lo referido al lugar de la conciencia en la definición de la identidad. En concreto, se señala que la identidad de una persona no es algo fijo, estable y unitario que se adquiere tempranamente en la vida. Más bien, es algo que está cambiando y, en tanto tal, es múltiple; la identidad está siendo construida y reconstruida continuamente en los encuentros con otras personas en el mundo. Esta es la justificación para señalar que la identidad es, en verdad, identidad social y, puntualmente, un constructo social. En esta línea, la idea de que la identidad es generada en instancias de interacción concreta y específica ha sido defendida por 
quienes enfatizan la naturaleza performativa de la misma. En este punto, Hyland sostiene que, toda vez que la identidad se pone en juego, fundamentalmente en la interacción social, está constreñida por la autoridad de la repetición histórica. Así, la idea de comunidad, desarrollada en el subtópico (2), es vital para entender los cruces entre disciplina e identidad. Detrás de cada compromiso individual con una profesión o área de conocimiento descansa una identidad institucional construida a través de inconmensurables interacciones. En este sentido, tanto la comunidad como la identificación colectiva con alguna de ellas sirven de ayuda no solo para comprender mejor el uso del lenguaje, sino también para apreciar las maneras en que aquella determina la constitución de la identidad. En (3), por último, el autor revisa la importancia del discurso en el acto de verbalizar una determinada posición que se asocia con un campo de estudio particular, y cómo esto, a su vez, conlleva un alienarse con las prácticas de su producción de conocimiento. De este modo, se definen los tópicos que se consideran como relevantes y, asimismo, se define el cómo hablar de ellos.

"Discipline: Proximity and positioning" es el nombre del segundo capítulo. En él, Hyland intenta dar forma a la idea de la conexión entre disciplina e identidad, esto, a través de la premisa de que la participación de los miembros de una comunidad científica en sus discursos académicos contribuye tanto a la cohesión disciplinaria como a la identidad individual. Para ello, se propone una matriz conceptual desde la cual abordar la tensión dilemática entre identidad y disciplina. Entendiendo que la identidad individual es un complejo único que combina diferentes experiencias grupales y personales, el autor sostiene que esta, además, se encuentra localmente construida a lo largo de dos "caminos discursivos", a saber: por un lado, a través de las relaciones entre "uno mismo" y la comunidad, lo que Hyland denomina proximidad, $\mathrm{y}$, por otro, mediante las relaciones entre el hablante y su mensaje, idea a la que refiere con el concepto de posicionamiento. A la proximidad le concierne el carácter interactivo del habla, representado por las convenciones sociales y discursivas de una disciplina (p. 43). En otras palabras, el uso del lenguaje está en directa relación con adoptar una voz disciplinaria que valide a un académico como alguien competente en su ámbito, de modo tal que pueda desplegar un self académico que lo vincule con los otros miembros de su comunidad. El posicionamiento, por su parte, se entiende como un puente entre la identidad y el discurso (p. 35), en términos de cómo las personas se sitúan discursivamente cuando interactúan con otros, esto fuertemente moldeado por los modos, más o menos establecidos, mediante los cuales se ve y se habla acerca del mundo en el marco de una comunidad académica. Esta, entonces, permearía la constitución de una identidad disciplinaria. De este modo, se enfatiza la idea de que las disciplinas corresponden, más bien, a comunidades culturales, y no a una simple designación institucional. Esta premisa ayuda a entender la mutua implicación entre los académicos y las comunidades a las que pertenecen, relación que cristaliza, a través de la interacción rutinaria, en formas discursivas que son indicativas de una identidad individual y grupal. 
En "Investigating identity", nombre del tercer capítulo, se pasa revisión a los métodos tradicionalmente utilizados para aproximarse al estudio de la identidad. En particular, tres son los principales modelos expuestos: el análisis conversacional, el análisis crítico del discurso y el análisis narrativo, cada uno de los cuales entiende el discurso como un campo donde emerge la identidad y, por ende, en donde las personas definen el quiénes son. Con todo, dada la importancia de la interacción en la conformación de identidades, los métodos empleados para su estudio deben procurar que la elaboración de los corpus se realice con base en ese carácter interactivo y dinámico. Así, indica Hyland, el análisis de estos develará la serie de rasgos y estrategias discursivas que predominan en las prácticas comunicativas de los académicos, así como determinados puntos de vista o posiciones respecto del conocimiento que se desarrolla en este tipo de comunidad de práctica. Una idea nuclear en este capítulo es aquella que se hace cargo del rol de los autores de los textos académicos. Mejor dicho: de la autoría de estos, siempre entendida como una práctica situada, cuyo proceso se lleva a cabo dentro de un marco relacional que tiene a los escritores, por un lado, y a los lectores, por otro, como sus elementos fundantes. Ambos se implican sinérgicamente. Atender a la complejidad de la autoría (a esa serie de rasgos experienciales que se conjugan en una subjetividad) es la tarea a la que se aboca Hyland en los capítulos sucesivos.

En el capítulo cuatro, "Identity in representational genres", el autor se propone verificar la ya mentada relación entre el self y los otros a través del análisis de lo que él denomina "géneros representacionales", es decir, tejidos discursivos mediante los cuales los académicos se representan a sí mismos, i. e., los agradecimientos de las tesis, las solicitudes de premios o becas para realizar tesis doctorales y las páginas de internet de académicos. Sus conclusiones apuntan a que, en los dos primeros géneros, ocurriría, de manera más clara, la emergencia de un "ethos disciplinario" o la construcción discursiva de una identidad disiciplinar que realiza constantes guiños a los valores presentes, en un momento particular, en la comunidad donde se producen. En ellos hay una autopromoción evidente. Esto se opone a lo que sucede con las páginas de internet, las que tienden a representar a los académicos en términos más genéricos, en tanto están dirigidas a audiencias más amplias y desconocidas. Particularmente, en este caso, se suscita la problemática sobre cómo se va definiendo la identidad disciplinaria en un contexto corporativo, y qué tipo de relaciones establecen las instituciones con sus académicos al presentarlos públicamente en internet.

En estrecho vínculo con lo anterior, en "Self-representation in academic bios", nombre del quinto capítulo, el autor lleva a cabo un análisis discursivo del género biografía académica. Sobre el supuesto de que este constituye "probably the most explicit public assertion of self-representation in scholarly life" [probablemente la afirmación de autorrepresentación pública más explícita en la vida académica] (p. 98, traducción nuestra), Hyland releva ciertas características presentes en 600 biografías de tres disciplinas, a saber, lingüística aplicada, ingeniería eléctrica y filosofía. En términos generales, su análisis sugiere la presencia de ciertas opciones 
representacionales provistas por y definidas desde la comunidad académica, de modo tal que las maneras en que puede instanciarse la identidad disciplinaria están más o menos constreñidas por el ámbito específico en el cual se insertan los autores. Así, las biografías de los ingenieros eléctricos dan gran importancia a los detalles educacionales y personales, mientras que las del área de filosofía enfatizan los intereses investigativos y las publicaciones. En un punto medio, en tanto, se ubicaría la biografía en lingüística aplicada.

Finalmente, y en línea con los capítulos previos, en "Culture: Authority and visibility", nombre del sexto apartado, se expone uno de los casos más interesantes respecto de la compleja conformación de una identidad disciplinaria. Aquí, Hyland analiza los reportes finales de las investigaciones realizadas por estudiantes de una universidad de Hong Kong. Una de las conclusiones más prominentes a la que llega el autor tiene que ver con las diferencias culturales manifiestas en el discurso académico de los estudiantes orientales, en contraste con los estadounidenses. Mientras los segundos hacen uso sistemático de pronombres autoriales y de la automención como mecanismos discursivos que sientan con fuerza la presencia de una voz autoral individual, los primeros se resisten a la marcación de la primera persona, pues supone, desde su perspectiva, una falta de solidaridad con el grupo al cual se pertenece. De esta manera, los estudiantes de Hong Kong privilegian una identidad colectiva por sobre una individual.

Sobre la base de que la producción de textos es siempre la producción de un self (p. 147), en el capítulo 7, "Reputation: Individuality and conformity", Hyland vuelve sobre los conceptos de proximidad y posicionamiento, es decir, la identidad construida a través de las relaciones entre los sujetos y sus comunidades, y entre los sujetos y sus mensajes, respectivamente. En esta línea, se pone de relieve el cómo las comunidades de prácticas van fijando ciertas convenciones retóricas para la divulgación de las identidades disciplinarias. En otras palabras, la institucionalidad adquiere un carácter normativo, en tanto constriñe las posibilidades discursivas de las identidades desplegadas, a la vez que proporciona las herramientas para esa comunicación y, más bien, para la ejecución exitosa de esa comunicación. Con todo, la argumentación sólida de las ideas y el lograr establecer un compromiso con los lectores parecen ser dos de los principales objetivos que guían la constitución de las identidades disciplinarias (p. 168).

Dentro de las múltiples opciones teórico-metodológicas que existen para aproximarse al estudio de la identidad, el concepto de género ha recibido una atenta y constante atención desde distintas disciplinas. En el capítulo 8, "Gender: Disciplinarity and positioning", Hyland plantea una interesante discusión acerca de la crítica feminista a la academia, un espacio históricamente dominado por hombres y que reproduce, naturalmente, lógicas propias de un sistema patriarcal. Esto, como marco teórico para un posterior análisis, a través de reseñas de libros (un género que posee un carácter fuertemente evaluativo, puesto que corresponde a la presentación, a la comunidad académica, de la investigación de otros), de eventuales diferencias de género. Las conclusiones apuntan a defender, en primer lugar, una 
visión interactiva y contextual tanto del género como de la identidad, alejándose, así, de las conceptualizaciones más esencialistas de estas. En segundo término, se asume que las maneras en que los hombres y las mujeres usan el lenguaje no están determinadas por el género, sino que se construyen, negocian y transforman en las prácticas sociales cotidianas en las que se dan encuentro los sujetos, definiendo, así, el género como una categoría eminentemente sociocultural.

Para finalizar, en "Identity, disciplinarity and methodology", nombre del noveno y último capítulo, el autor provee una exposición que vuelve sobre los elementos nucleares de su libro, desde su intento por vincular (y problematizar) la identidad con la comunidad académica, hasta sus propuestas metodológicas para llevar a cabo un examen de este par dilemático en los términos que él intenta defender a lo largo de su obra, es decir, la identidad y la academia vistas como categorías fuertemente arraigadas al contexto y emergentes en la interacción.

En esta reseña nos propusimos sintetizar los aspectos más relevantes de las proposiciones enunciadas por Hyland. En este sentido, nos parece que la exposición de las ideas, además de ser absolutamente clara y precisa, da cuenta de un posicionamiento teórico-metodológico que se alinea con los desarrollos postestructuralistas del estudio de la identidad, toda vez que le otorga un lugar prominente a la interacción como marco dentro del cual esta emerge y se despliega. Asimismo, su trabajo se sustenta sobre una importante evidencia empírica a través del análisis de géneros discursivos propios del ámbito académico, de modo tal que las distintas ideas propuestas se ven respaldadas por los datos. En esta línea, junto con celebrar la propuesta de Hyland, creemos que constituiría un valioso aporte a la comunidad académica, en general, y al estado actual de los estudios sobre identidad, en particular, llevar a cabo una pesquisa que replique, en algún nivel, este trabajo en la academia chilena.

En conclusión, como se ha intentado poner de manifiesto, la superación del entendimiento de la identidad (y de la identidad disciplinaria, concretamente) como una categoría estanca y prediscursiva, y su abordaje desde una óptica dinámica y social, no hace más que destacar su naturaleza creativa, siempre hacia un otro e histórica, en nomenclatura coseriana.

CRISTIAN Rojas InOSTROZA

Universidad de Chile rojascristian@ug.uchile.cl 